\title{
Association Between Vitamin D Levels and Treatment Response to Direct-Acting Antivirals in Chronic Hepatitis C: A Real-World Study
}

\author{
Vijay Gayamª, c, Amrendra Kumar Mandala , Mazin Khalid ${ }^{\mathrm{a}}$, Osama Mukhtara, Arshpal Gilla , \\ Pavani Garlapati ${ }^{\mathrm{a}}$, Benjamin Tiongson ${ }^{\mathrm{a}}$, Jagannath Sherigar ${ }^{\mathrm{b}}$, \\ Mohammed Mansour ${ }^{\mathrm{a}}$, Smruti Mohanty ${ }^{\mathrm{b}}$
}

\begin{abstract}
Background: Low serum vitamin D levels in chronic hepatitis C (CHC) is associated with advanced liver fibrosis; and there remains an imprecise relationship with the treatment response based on the vitamin D levels. Previous studies have shown conflicting results on the vitamin D levels, and association with treatment response in $\mathrm{CHC}$ treated with interferon-based regimens.
\end{abstract}

Methods: Patients with $\mathrm{CHC}$ treated with direct-acting antivirals (DAAs) between January 2016 and December 2017 in the community clinic setting were retrospectively analyzed. Pretreatment baseline patient characteristics, treatment efficacy with the sustained virologic response at 12 weeks post-treatment (SVR 12) were assessed in CHC patients with deficient, insufficient, and normal levels of vitamin D measured before the initiation of DAA therapy.

Results: Two hundred and ninety-one patients were included in the study. Direct-acting antivirals included in the study were ledipasvir/ sofosbuvir \pm ribavirin, ombitasvir + paritaprevir + ritonavir + dasabuvir \pm ribavirin, and sofosbuvir/velpatasvir. An overall sustained virologic response was achieved in $95 \%(n=276)$ of patients. SVR 12 rates among patients with vitamin D deficiency, vitamin D insufficiency and normal vitamin D levels were $92 \%, 96.2 \%$, and $97.2 \%$ respectively and was not statically significant $(P=0.214)$. A total of 71 patients were cirrhotic. The prevalence of vitamin D insufficiency (20 - $29.9 \mathrm{ng} / \mathrm{mL})$ and deficiency $(<20 \mathrm{ng} / \mathrm{mL})$ was significantly higher in cirrhotic patients $(\mathrm{P}=0.01)$. Despite this, pretreatment vitamin $\mathrm{D}$ levels did not show any impact on the virologic response. The most common adverse effect observed was fatigue. None of the patients had to discontinue the treatment due to adverse events.

Manuscript submitted July 12, 2018, accepted July 23, 2018

aDepartment of Medicine and Gastroenterology, Interfaith Medical Center, 1545 Atlantic Avenue. Brooklyn, NY 11213, USA

bepartment of Medicine and Division of Gastroenterology and Hepatology, New York-Presbyterian Brooklyn Methodist Hospital, 506, 6th St, Brooklyn, NY 11215, USA

${ }^{\mathrm{c}}$ Corresponding Author: Vijay Gayam, Interfaith Medical center, 1545 Atlantic Ave, Brooklyn, NY 11213, USA. Email: vgayam@interfaithmedical.com

doi: https://doi.org/10.14740/gr1072w
Conclusions: DAAs are safe and effective with a high overall SVR 12 in $\mathrm{CHC}$ and treatment response does not depend on the pretreatment vitamin D levels. The prevalence of both vitamin D insufficiency and deficiency was observed to be higher in cirrhotic cohorts compared to non-cirrhotic counterparts.

Keywords: Chronic hepatitis C; Vitamin D level; Sustained virologic response; Cirrhosis

\section{Introduction}

About 71 million people are chronically infected with hepatitis $\mathrm{C}$ virus (HCV) and carry an elevated risk of developing liver disease including liver cirrhosis and hepatocellular carcinoma (HCC) [1]. HCV related deaths are approximated to be 399,000 annually [2]. Consequently, eradication of $\mathrm{HCV}$ is an essential endpoint as effective treatment has been associated with the reduction of hepatic fibrosis and decreased rates of $\mathrm{HCC}[3,4]$.

Vitamin D is known to have regulatory roles in several infections as an immune modulator in both innate and adaptive immunity, principally via regulatory pathways of T-cell function [5-8]. Vitamin D deficiency is a very common observation among patients with the chronic liver disease, with nearly one third of these patients having severe vitamin D deficiency $(<$ $12 \mathrm{ng} / \mathrm{mL}$ ) [9]. This may be attributable to the impaired physiological conversion of inactive vitamin $\mathrm{D}$ to biologically active metabolites in chronic liver disease, which in turn is associated with severe fibrosis and reduced responsiveness to previously used interferon-based therapy [9].

Several studies have evaluated the role of vitamin D deficiency concerning interferon-based therapies, but there remains a paucity of literature which examines the influence of vitamin D levels with direct-acting antivirals (DAAs) response rates $[10,11]$. We aim to assess the predictive value of pretreatment serum vitamin D level for achieving sustained virologic response at 12 weeks post-treatment (SVR 12), defined as an undetectable viral load 12 weeks post-therapy. Secondary objectives included evaluating the prevalence of serum 25-vitamin D levels in HCV related cirrhotic cohorts. 


\section{Patients and Methods}

The Institutional Review Board (IRB) approved the study protocol, and the patients were recruited from two specialty clinics attached to the two large community hospitals: Interfaith Medical Center and New York-Presbyterian Brooklyn Methodist Hospital.

\section{Patients}

A total of 291 consecutive chronic HCV infected patients treated with DAAs between January 2016 and December 2017 in the community clinic setting were retrospectively analyzed.

Decompensated cirrhosis, hepatocellular carcinoma, history of liver transplant, and human immunodeficiency virus (HIV) co-infection were excluded from the study. None of the patients included in this study discontinued the treatment due to adverse events associated with treatment medications.

The 25-hydroxyvitamin D levels were assessed before the start of therapy with chemiluminescence immunoassay; concentrations were recorded in $\mathrm{ng} / \mathrm{mL}$. Vitamin $\mathrm{D}$ deficiency was defined as a $25(\mathrm{OH})$-vitamin $\mathrm{D}$ serum level $<20 \mathrm{ng} / \mathrm{mL}$, vitamin D insufficiency as $25(\mathrm{OH})$-vitamin D levels of $20-29.9$ $\mathrm{ng} / \mathrm{mL}$, and normal vitamin D levels $\geq 30 \mathrm{ng} / \mathrm{mL}$ [12].

All patients in this retrospective cohort study received at least 12 weeks of treatment with one of the recommended combination regimens in standard doses for chronic HCV infection. Three different treatment regimens were included in our study: ledipasvir (LDV) $90 \mathrm{mg} /$ day + sofosbuvir (SOF) $400 \mathrm{mg} /$ day , ledipasvir $90 \mathrm{mg} /$ day + sofosbuvir $400 \mathrm{mg} /$ day + ribavirin $(\mathrm{RBV}) 1,000 \mathrm{mg} /$ day if $<75 \mathrm{~kg}$ and $1,200 \mathrm{mg} /$ day if $\geq 75 \mathrm{~kg}$, (ombitasvir $12.5 \mathrm{mg}+$ paritaprevir $75 \mathrm{mg}+$ ritonavir $50 \mathrm{mg}$ ) two tablets twice daily + dasabuvir $250 \mathrm{mg}$ twice daily, ombitasvir (OBV) 12.5mg + paritaprevir (PTV) $75 \mathrm{mg}$ + ritonavir (r) $50 \mathrm{mg}$ two tablets twice daily plus dasabuvir (DSV) $250 \mathrm{mg}$ twice daily + ribavirin $1,000 \mathrm{mg} /$ day if $<75$ $\mathrm{kg}$ and $1,200 \mathrm{mg} /$ day if $\geq 75 \mathrm{~kg}$, and sofosbuvir $400 \mathrm{mg} /$ day + velpatasvir (VEL) $400 \mathrm{mg} /$ day. Duration of treatment period ranged from 12 weeks $(n=250)$ to 24 weeks $(n=41)$. The regimens selection was based on the American Association for the Study of Liver Disease (AASLD) guidelines [13].

\section{Study assessments}

Treatment efficacy, safety, and tolerability were assessed by reviewing documented adverse events, treatment completion rates, any dosage reductions, and discontinuation of medications. Pre-treatment laboratory studies were compared with the post-treatment studies to identify any significant lab abnormalities associated with antiviral agents. The diagnosis of liver cirrhosis was based on a combination of clinical symptoms, laboratory parameters including FibroSure score $\geq 0.75$, imaging modalities (USG and/or CT scan) and histopathology when indicated. We included compensated cirrhosis, defined as the presence of cirrhosis but the absence of ascites, jaundice, hepatic encephalopathy and variceal bleeding, as defined by the
AASLD guidelines.

Treatment response was assessed with HCV RNA viral load (IU/ $\mathrm{mL}$ ) at 4 weeks after initiation of treatment, at the end of treatment, and 12 weeks after completion of therapy. The test was performed using COBAS $\AA$ AmpliPrep/COBAS $\AA$ TaqMan® HCV Quantitative Test, v2.0 (Roche molecular diagnostics) with the lower limit of quantification (LLOQ) of HCV RNA $15 \mathrm{IU} / \mathrm{mL}$. HCV genotyping was assessed by polymerase chain reaction (PCR) amplification and solid-phase electrochemical detection. SVR 12 was defined as the undetectable viral load at 12 weeks after the end of treatment.

\section{Statistical analysis}

The SPSS ${ }^{\circledR}$ statistics software package (IBM SPSS ${ }^{\circledR}$ statistics version 21, USA) was used for statistical analysis. Values were expressed as mean \pm SD and mean categorical values were analyzed using Student's $t$-test. Differences in categorical values were analyzed by Chi-square test. All P-values were two-tailed and $\mathrm{P} \leq 0.05$ was considered significant. One-way analysis of variance (ANOVA) was used to determine whether there were differences among the group means. Multivariable logistic regression was performed only in variables with a $\mathrm{P} \leq 0.05$ in univariate analysis.

\section{Results}

\section{Baseline characteristics}

Baseline characteristics are shown in Table 1. Mean age of the patients in the study of the cohort was $60.35 \pm 10.4$ years. Majority of the patients were male $179(61.5 \%)$. Genotype 1a was the predominant $\mathrm{HCV}$ genotype $(52.1 \%)$, followed by genotype $1 \mathrm{~b}$ (30.1\%), genotype $2(5.5 \%)$, genotype $3(4.1 \%)$, and genotype $4(2 \%)$. Seventy-one patients $(24.4 \%)$ had compensated cirrhosis. Two hundred and thirty-one $(79.4 \%)$ were treatment-naive patients, and $60(20.6 \%)$ were treatment-experienced with interferon-based regimens. Ninety-four patients $(32.3 \%)$ had a history of diabetes, 146 had hypertension $(50.2 \%), 26$ had coronary artery disease (CAD), and $25(8.6 \%)$ patients had chronic kidney disease (CKD). None of the patients had decompensated cirrhosis, hepatocellular carcinoma, or a previous liver transplant.

Patients were divided into three groups based on pretreatment vitamin D levels. Vitamin D deficiency included 113 patients, vitamin D insufficiency included 105 patients, and normal vitamin D level included 73 patients. The three groups were similar in their baseline characteristics except for cirrhosis and bilirubin levels. The vitamin D insufficiency and vitamin $\mathrm{D}$ deficiency groups both had a higher incidence of cirrhotic patients $(\mathrm{P} \leq 0.001)$ and elevated levels of bilirubin $(\mathrm{P}=0.012)$.

\section{Treatment regimens and overall SVR 12 achievement}

Among the 291 patients, 184 patients $(63.2 \%)$ were in the $\mathrm{SOF} / \mathrm{LDV}$ group, $59(20.3 \%)$ in the OBV/PTV/r + DSV group 
Table 1. Demographic and Clinical Characteristics of Patients at Baseline With Treatment Regimen

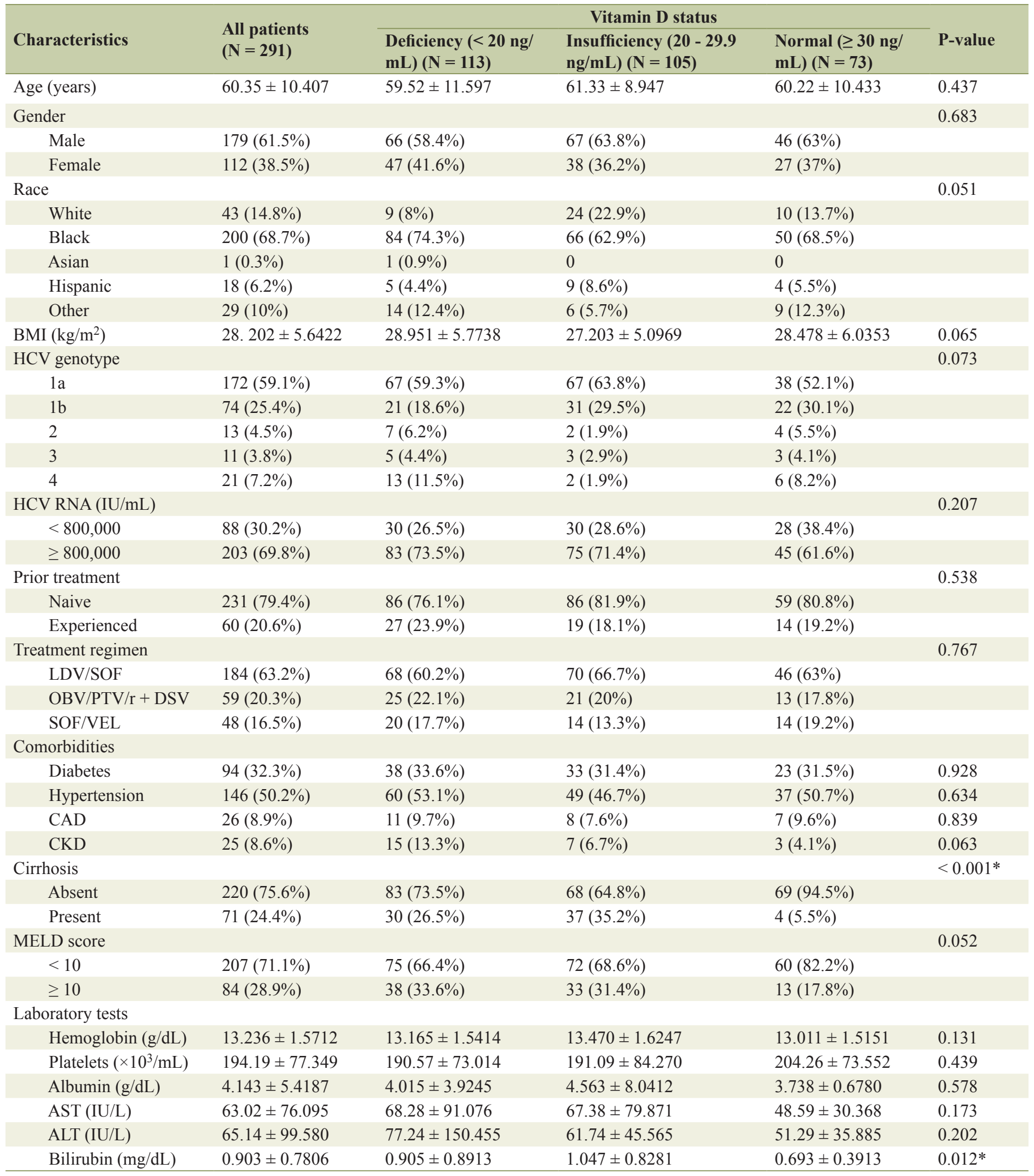

Data presented as number/total number (percent). *Statistically significant P-values. BMI: body mass index; HCV: hepatitis C virus; RNA: ribonucleic acid; MELD: model for end-stage liver disease; ALT: alanine transaminase; AST: aspartate aminotransferase; CAD: coronary artery disease; CKD: chronic kidney disease. 
Table 2. Demographic and Clinical Characteristics of Patients at Baseline by Treatment Response

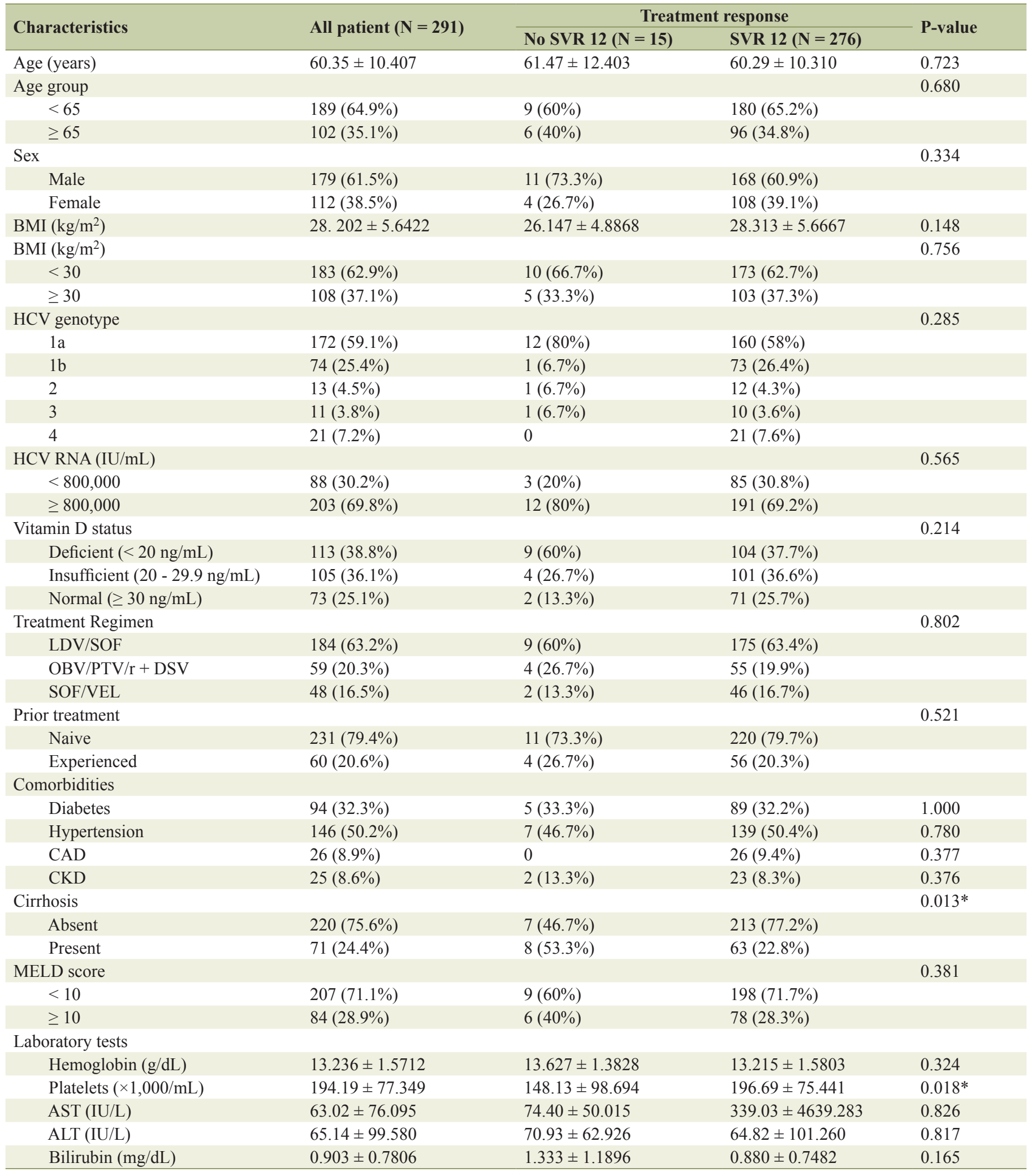

Data presented as number/total number (percent). *Statistically significant P-values. BMI: body mass index; HCV: hepatitis C virus; RNA: ribonucleic acid; MELD: model for end-stage liver disease; ALT: alanine transaminase; AST: aspartate aminotransferase; CAD: coronary artery disease; CKD: chronic kidney disease. 
Table 3. SVR 12 Rate in Patients Based on Vitamin D levels

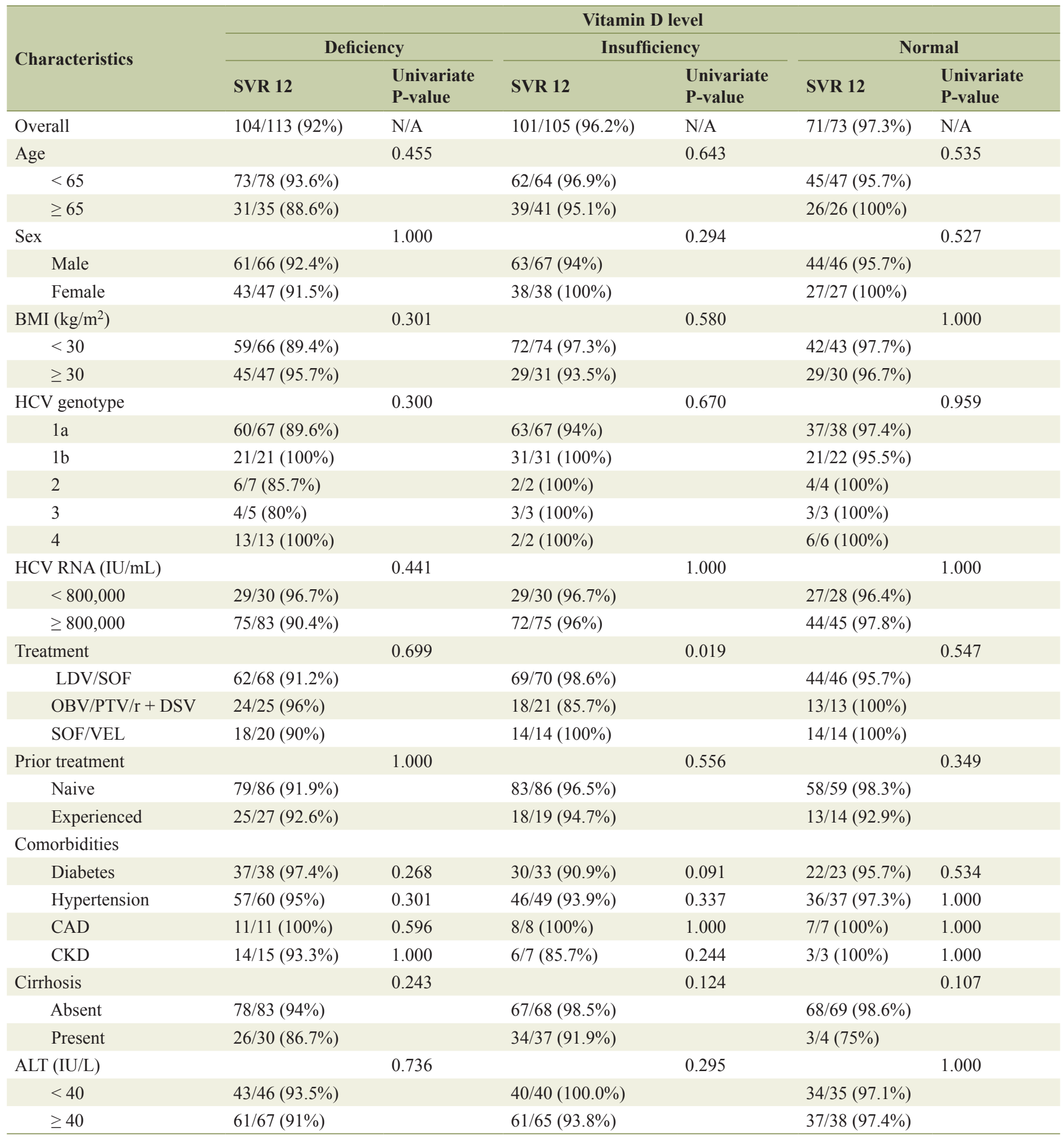

Data presented as number/total number (percent). BMI: body mass index; HCV: hepatitis C virus; RNA: ribonucleic acid; MELD: model for end-stage liver disease; ALT: alanine transaminase; CAD: coronary artery disease; CKD: chronic kidney disease.

and $48(16.5 \%)$ patients in the SOF/VEL group.

In order to identify the factors associated with the treatment failure, the univariate and multivariate analysis was performed.
Patients were divided into two subgroups based on treatment response, which were the No SVR 12 group and the SVR 12 group. SVR 12 in entire cohort was $94.8 \%$, and showed no 
Table 4. Association of Vitamin D Levels With Cirrhosis

\begin{tabular}{lllll}
\hline \multirow{2}{*}{ Vitamin D status } & \multicolumn{2}{c}{ Cirrhosis } & Total $(\mathbf{N}=\mathbf{2 9 1})$ & P-value \\
\cline { 2 - 4 } & Absent $(\mathbf{N}=\mathbf{2 2 0})$ & Present $(\mathbf{N}=71)$ & $22.830 \pm 8.4601$ & 0.006 \\
\hline All patients & $23.452 \pm 9.0978$ & $20.901 \pm 5.7075$ & $113(60.8 \%)$ & $<0.001$ \\
Deficiency $(<20 \mathrm{ng} / \mathrm{mL})$ & $83(54.6 \%)$ & $30(88.2 \%)$ & $105(59 \%)$ & $<0.001$ \\
Insufficiency $(20-29.9 \mathrm{ng} / \mathrm{mL})$ & $68(49.6 \%)$ & $37(90.2 \%)$ & 2 \\
\hline
\end{tabular}

Data presented as number/total number (percent).

statistically significant associations with vitamin D level, age, race, body mass index (BMI), HCV genotype, HCV viral load, $\mathrm{HCV}$ prior treatment status, HCV treatment regimen, model for end-stage liver disease (MELD) score, baseline aspartate aminotransferase (AST), baseline alanine aminotransferase (ALT), medical co-morbidities, baseline hemoglobin.

A statistically significant difference was noted with cirrhosis status $(\mathrm{P}=0.013)$ and baseline platelet counts $(\mathrm{P}=0.018)$ (Table 2). After adjusting the variables and baseline characteristics in the multivariate analysis, these findings remained consistent. For cirrhosis and baseline platelet counts multivariate P-values were 0.012 and 0.017 respectively.

\section{Vitamin D level and virologic response}

SVR 12 rates among patients with vitamin D deficiency, vitamin D insufficiency and normal vitamin D levels were $92 \%$, $96.2 \%$, and $97.2 \%$ respectively. SVR 12 did not show statistically significant associations with age, BMI, HCV genotype, $\mathrm{HCV}$ viral load, HCV prior treatment status, HCV treatment regimen, cirrhosis status, MELD score, baseline AST, ALT, baseline hemoglobin or medical co-morbidities (Table 3).

\section{Cirrhosis and pretreatment vitamin D levels}

A total of 71 patients had liver cirrhosis in the study popu- lation; 30 patients were in vitamin D deficiency group, and 37 were in vitamin D insufficiency group. There was no significant difference in pretreatment mean level of vitamin $\mathrm{D}$ in patients with and without cirrhosis $(20.90 \pm 5.70$ versus 23.45 $\pm 9.09 \mathrm{ng} / \mathrm{mL}$ ). However, more cirrhotic patients had vitamin $\mathrm{D}$ insufficiency and deficiency compared to the non-cirrhotic patient $(\mathrm{P} \leq 0.001)$ (Table 4$)$.

\section{Adverse effects}

The most common adverse effect is fatigue seen in $81(27.8 \%)$ patients across all vitamin $\mathrm{D}$ groups. The other adverse effects were anemia $41(14.1 \%)$ followed by arthralgia 12(4.1\%), headache $15(5.2 \%)$. There were no severe AEs observed in the entire cohort. All patients tolerated treatment well (Table 5).

\section{Discussion}

Our study gives a real-world experience of association between the vitamin D levels and the treatment response to DAAs. To our knowledge, the current study represents the largest studied cohort in published literature to date after the availability of the DAA regimens in the community care setting. Existing clinical studies have shown an excellent response to DAAs in HCV infection [14]. Our study findings are also consistent with the clinical studies with an overall SVR 12 rate of $94.8 \%$ and treat-

Table 5. Adverse Effects

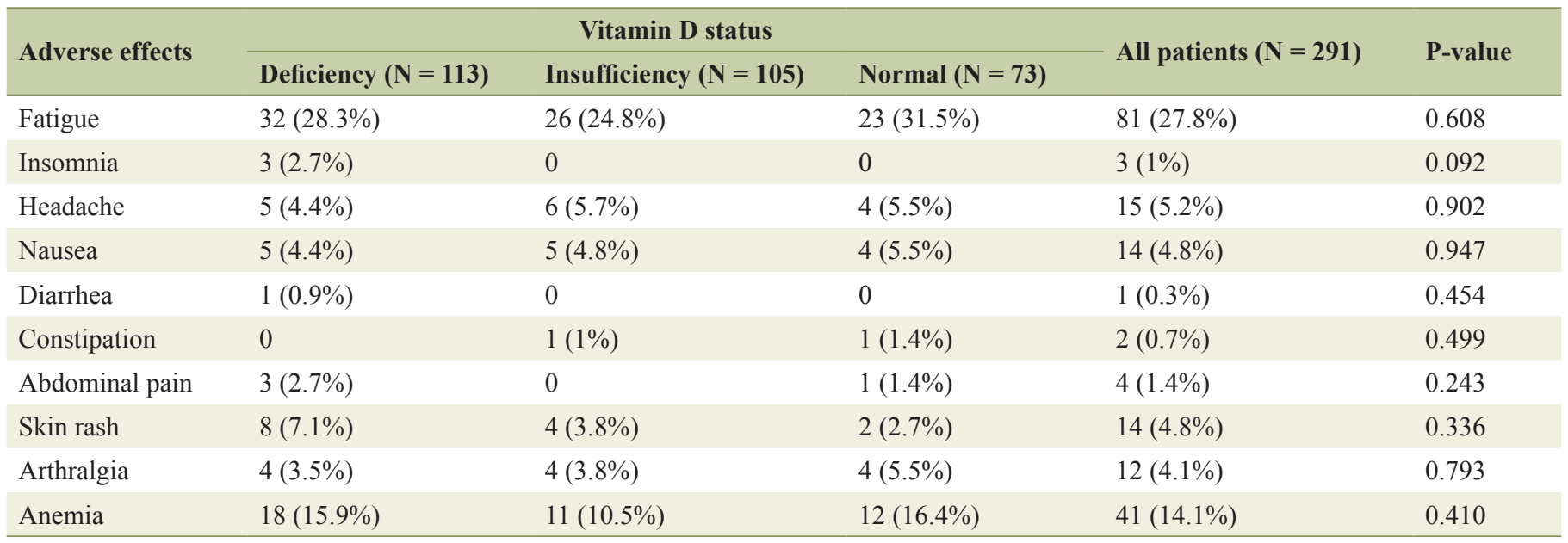

Data presented as number (percent). 
ment response did not differ based on vitamin D levels.

Petta et al retrospectively analyzed the relationship of vitamin D levels with peg-interferon/ribavirin for hepatitis C and reported an association between lower vitamin $\mathrm{D}$ serum levels and failure to achieve SVR 12 [15]. However, two previous meta-analyses have evaluated associations of vitamin $\mathrm{D}$ and treatment outcomes in the context of interferon-based therapy with conflicting results $[16,17]$.

Backstedt et al evaluated the role of vitamin D levels on SVR 12 with interferon-based regimens and non-interferon based DAAs. Our study findings are consistent with their study, where pre-treatment vitamin D levels were not associated with SVR 12. A key difference in our study is the higher SVR 12 rate of $94.5 \%$ vs. SVR 12 rate of $78 \%$ in their study [18]. Comparing SVR 12 between cirrhotics and non-cirrhotics groups, Backstedt et al reported cirrhotic patients had SVR 12 rates of $71 \%$ versus $96 \%$ in non-cirrhotic which is again higher in our study with SVR of $86.7 \%$ in cirrhotics vs. 94 $\%$ in non-cirrhotics. The difference in the treatment response could be due to the inclusion of interferon-based regimens in that study.

With the advent of newer DAAs, there are increased SVR 12 rates in clinical practice, further lessening the role of vitamin D supplementation specifically to augment the response. DAA therapy is a targeted approach where the treatment is less dependent on host immune factors, unlike peg-interferon and ribavirin $[19,20]$.

There is emerging data showing vitamin D deficiency being associated with advanced degrees of fibrosis in chronic HCV infection [21]. We also observed a high prevalence of vitamin $\mathrm{D}$ deficiency in our study population with the significantly higher number of deficiency and insufficiency of vitamin D levels in the cirrhotic subgroup, which is consistent with the published literature [18, 22]. Putz-Bankuti et al and Baur et al also observed that low levels of $25(\mathrm{OH})$-vitamin $\mathrm{D}$ are associated with fibrosis and hepatic decompensation and death as well [23, 24].

DAAs included in our study were generally well tolerated, and their adverse events were consistent with other DAA based studies in the literature [14]. Common adverse effects were fatigue, headache, nausea, arthralgia, and rash. Additionally, anemia was noted in patients who were taking DAA concurrently with ribavirin. None of the patients in the study discontinued therapy due to any adverse event.

Our study is unique in assessing the association between the vitamin D levels and the treatment response to DAAs in $\mathrm{CHC}$ in a real-world community setting. This study reiterates the prevalence of vitamin D deficiency in the cirrhotic patients with $\mathrm{HCV}$ infection and will guide the clinicians effectively treat $\mathrm{CHC}$ with vitamin D insufficiency and deficiency. While our study revealed that in newer DAA therapy, vitamin D levels do not influence SVR 12, further studies are still warranted to evaluate optimal vitamin D levels in patients with cirrhosis, as vitamin D supplementation may still be of benefit due to pleiotropic effects on multiple organ systems [19, 20]. Larger randomized trials are necessary to derive more robust conclusions. Some of the limitations of the present study include a retrospective design and changes in vitamin $\mathrm{D}$ levels were not measured during the treatment period and after the treatment.

\section{Conclusions}

Based on our findings, we observed the high prevalence of vitamin D deficiency in cirrhotic populations. Despite this, pretreatment vitamin D level did not impact treatment response. DAAs are safe and effective with a high overall sustained virologic response.

\section{Conflict of Interest}

Dr. Mohanty is on the Speakers Bureau for Gilead Science, BMS, and Abbvie Pharmaceuticals. For the remaining authors, there is no conflict of interest.

\section{Funding}

None.

\section{Author Contributions}

Vijay Gayam: substantial contribution to conception and design of the study, data acquisition, analysis, interpretation of the data and drafting of the manuscript; and performed critical reviews, communicated with the journal, and addressed comments from the reviewers. Amrendra Kumar Mandal and Mazin Khalid: data acquisition and provided assistance with interpretation of data and drafting of the manuscript. Osama Mukhtar: substantial contribution to statistical data analysis and interpretation of the data. Pavani Garlapati, Tiongson Benjamin, and Arshpal Gill have contributed in the acquisition of the patient data. Sherigar Jagannath, Mohammad Mansour, and Smruti Mohanty did critical review of the manuscript. All the authors vouch for the veracity and completeness of the data presented and agreed to submit the manuscript for publication.

\section{Abbreviations}

DAAs: direct-acting antivirals; AE: adverse event; HCV: hepatitis C virus; HCC: hepatocellular cancer; GT: genotype; SVR12: sustained virologic response at 12 weeks post-treatment; LDV: ledipasvir; SOF: sofosbuvir; VEL: velpatasvir; RBV: ribavirin; OBV: ombitasvir; PTV/r: paritaprevir/ritonavir; DSV: dasabuvir

\section{References}

1. Retrieved from http://www.who.int/news-room/factsheets/detail/hepatitis-C.

2. Mohd Hanafiah K, Groeger J, Flaxman AD, Wiersma ST. Global epidemiology of hepatitis $\mathrm{C}$ virus infection: new estimates of age-specific antibody to HCV seroprevalence. Hepatology. 2013;57(4):1333-1342.

3. Butt AA, Wang X, Moore CG. Effect of hepatitis C virus 
and its treatment on survival. Hepatology. 2009;50(2):387392.

4. Morgan RL, Baack B, Smith BD, Yartel A, Pitasi M, Falck-Ytter Y. Eradication of hepatitis $\mathrm{C}$ virus infection and the development of hepatocellular carcinoma: a meta-analysis of observational studies. Ann Intern Med. 2013;158(5 Pt 1):329-337.

5. DeLuca HF. Overview of general physiologic features and functions of vitamin D. Am J Clin Nutr. 2004;80(6 Suppl):1689S-1696S.

6. Muhe L, Lulseged S, Mason KE, Simoes EA. Casecontrol study of the role of nutritional rickets in the risk of developing pneumonia in Ethiopian children. Lancet. 1997;349(9068):1801-1804.

7. Lin R, White JH. The pleiotropic actions of vitamin D. Bioessays. 2004;26(1):21-28.

8. Muller K, Bendtzen K. 1, 25-Dihydroxyvitamin D3 as a natural regulator of human immune functions. J Investig Dermatol Symp Proc. 1996;1(1):68-71.

9. Arteh J, Narra S, Nair S. Prevalence of vitamin D deficiency in chronic liver disease. Dig Dis Sci. 2010;55(9):26242628.

10. Bitetto D, Fabris C, Fornasiere E, Pipan C, Fumolo E, Cussigh A, Bignulin S, et al. Vitamin D supplementation improves response to antiviral treatment for recurrent hepatitis C. Transpl Int. 2011;24(1):43-50.

11. Abu-Mouch S, Fireman Z, Jarchovsky J, Zeina AR, Assy N. Vitamin D supplementation improves sustained virologic response in chronic hepatitis $\mathrm{C}$ (genotype 1)-naive patients. World J Gastroenterol. 2011;17(47):5184-5190.

12. Holick MF, Binkley NC, Bischoff-Ferrari HA, Gordon CM, Hanley DA, Heaney RP, Murad MH, et al. Evaluation, treatment, and prevention of vitamin D deficiency: an Endocrine Society clinical practice guideline. J Clin Endocrinol Metab. 2011;96(7):1911-1930.

13. Retrieved from https://www.aasld.org/about-aasld/pressroom/hcv-guidance-updates-recommendations-screening-and-treating-key-populations.

14. Falade-Nwulia O, Suarez-Cuervo C, Nelson DR, Fried MW, Segal JB, Sulkowski MS. Oral direct-acting agent therapy for hepatitis $\mathrm{C}$ virus infection: a systematic review. Ann Intern Med. 2017;166(9):637-648.
15. Petta S, Camma C, Scazzone C, Tripodo C, Di Marco V, Bono A, Cabibi D, et al. Low vitamin D serum level is related to severe fibrosis and low responsiveness to interferon-based therapy in genotype 1 chronic hepatitis $\mathrm{C}$. Hepatology. 2010;51(4):1158-1167.

16. Kitson MT, Dore GJ, George J, Button P, McCaughan GW, Crawford DH, Sievert W, et al. Vitamin D status does not predict sustained virologic response or fibrosis stage in chronic hepatitis $\mathrm{C}$ genotype 1 infection. J Hepatol. 2013;58(3):467-472.

17. Garcia-Alvarez M, Pineda-Tenor D, Jimenez-Sousa MA, Fernandez-Rodriguez A, Guzman-Fulgencio M, Resino S. Relationship of vitamin D status with advanced liver fibrosis and response to hepatitis $\mathrm{C}$ virus therapy: a metaanalysis. Hepatology. 2014;60(5):1541-1550.

18. Backstedt D, Pedersen M, Choi M, Seetharam A. 25-Vitamin D levels in chronic hepatitis C infection: association with cirrhosis and sustained virologic response. Ann Gastroenterol. 2017;30(3):344-348.

19. Holick MF. Vitamin D deficiency. N Engl J Med. 2007;357(3):266-281.

20. Rahman AH, Branch AD. Vitamin D for your patients with chronic hepatitis C? J Hepatol. 2013;58(1):184-189.

21. White DL, Tavakoli-Tabasi S, Kanwal F, Ramsey DJ, Hashmi A, Kuzniarek J, Patel P, et al. The association between serological and dietary vitamin $\mathrm{D}$ levels and hepatitis C-related liver disease risk differs in African American and white males. Aliment Pharmacol Ther. 2013;38(1):28-37.

22. Iruzubieta P, Teran A, Crespo J, Fabrega E. Vitamin D deficiency in chronic liver disease. World J Hepatol. 2014;6(12):901-915.

23. Putz-Bankuti C, Pilz S, Stojakovic T, Scharnagl H, Pieber TR, Trauner M, Obermayer-Pietsch B, et al. Association of 25-hydroxyvitamin D levels with liver dysfunction and mortality in chronic liver disease. Liver Int. 2012;32(5):845-851.

24. Baur K, Mertens JC, Schmitt J, Iwata R, Stieger B, Eloranta JJ, Frei P, et al. Combined effect of $25-\mathrm{OH}$ vitamin D plasma levels and genetic vitamin D receptor (NR 1I1) variants on fibrosis progression rate in $\mathrm{HCV}$ patients. Liver Int. 2012;32(4):635-643. 